Rev. Biol. Neotrop. 10(1): 09-20. 2013

\title{
STRUTURA DA COMUNIDADE DE ALGAS PERIFÍtICAS EM DISTINTAS Veredas no Cerrado goiano
}

\author{
Josimeire Aparecida Leandrini \\ Universidade Federal Fronteira Sul (UFFS). Pós-graduação em Agroecologia e Desenvol- \\ vimento Rural Sustentável. Campus Laranjeiras do Sul, PR. Email: jaleandri@gmail.com
}

\section{Sirlene Aparecida Felisberto}

Universidade Federal de Goiás (UFG). Programa de Pós-Graduação em Biodiversidade Vegetal, Instituto de Ciências Biológicas. Campus Samambaia, Goiânia-GO. Email: fsirfe@gmail.com

\section{Bárbara DUNCK}

Universidade Estadual de Maringá (UEM). Programa de Pós-Graduação em Ecologia de Ambientes Aquáticos Continentais (PEA-UEM). Email: dunck.barbara@gmail.com

\begin{abstract}
Resumo: Impactos ambientais proporcionados por ação antrópica alteram o desenvolvimento das comunidades biológicas. Por isso, o presente estudo objetivou comparar os atributos estruturais de comunidades de algas perifíticas em Veredas sob diferentes impactos antrópicos. Para o estudo, seis Veredas localizadas na região sudeste do Estado de Goiás foram selecionadas, as quais estão subdivididas em duas conservadas, duas impactadas por agropecuária e duas em área urbana. As variáveis bióticas e abióticas foram obtidas em agosto e setembro de 2008; abril e maio de 2009; março de 2010. A sazonalidade foi um fator importante na distinção das Veredas quanto às características limnológicas. Veredas em período de seca (2008) apresentaram menores concentrações de fósforo e maior turbidez, enquanto que em período chuvoso (2010) foram relacionadas com pH mais próximo da neutralidade. Maiores valores de densidade algal foram registrados para Vereda urbana, com predomínio de Bacillariophyceae em setembro de 2008 e Cyanophyceae em maio de 2009. Entre os 15 táxons detectados como espécies indicadoras, 11 foram relacionados com Veredas em áreas de agropecuária e urbana. As diferenças na densidade da comunidade de algas perifíticas nas seis Veredas foram determinadas principalmente pela maior disponibilidade de nutrientes e turbidez mais elevada. Concluímos que os maiores valores de riqueza de espécies, diversidade e densidade de algas perifíticas de Veredas impactadas devem-se a fatores proporcionados pela ação antrópica e a sazonalidade.
\end{abstract}

Palavras-chave: Águas ácidas, ecologia, epifíton, solos hidromórficos.

Abstract: Environmental impacts provided by human actions alter the development of biological communities. This study aimed to evaluate the changes in structural attributes of periphytic algae in wetlands, among two conserved, two impacted by agriculture and two impacted in an urban area. The six Palm swamps are located in the Southeastern region of the Goiás state. Biotic and abiotic data were obtained in August and September 2008, April and May 2009, March 2010. Seasonality was an important factor in distinguishing in the limnological characteristics of the wetlands. Palm swamps in the dry period (2008) showed lower concentrations of phosphorus and greater turbidity, while in the rainy season (2010) were connected with pH closer to neutrality. Higher values of algal density were recorded for urban Palm swamps, predominantly the Bacillariophyceae in September 2008 and Cyanophyceae in May 2009. Among the 15 taxa selected as indicator species 11 were related to Palm swamps in agriculture and urban areas. Changes in the community density of periphytic algae in the six Palm swamps were mainly determined to the increased availability of nutrients and turbidity. We conclude that the higher values of species richness, diversity and density of periphytic algae Palm swamps impacted should to factors provided by action anthropogenic and seasonality.

KEY-wORDs: acidic water, epiphyton ecology, hidromorphic soils. 


\section{INTRODUÇÃO}

Cerrado compreende um mosaico de formações vegetais que variam desde campos abertos até formações densas de florestas (Eiten, 1972, 1994; Ribeiro \& Walter, 1998). Dentre os tipos fitofisionômicos do Cerrado, as Veredas são caracterizadas por possuírem solos hidromórficos saturados por água a maior parte do ano e por apresentarem nascentes que formam cursos d’água estacionais e/ou permanentes (Ferreira, 2003). Nesse sentido tais fisionomias funcionam como um filtro que regulam o fluxo de água, sedimentos e nutrientes de terrenos mais altos da bacia hidrológica (Lima, 1991).

Vários são os fatores impactantes que se estendem às Veredas: desmatamento e empobrecimento genético; represamento dos cursos d'água; degradação dos solos; contaminação química e física da água e da biota; irrigação; implantação e construção de estradas; exploração de recursos minerais (Ferreira, 2003).

Dentre os componentes bióticos que recebem influência dos contaminantes nas Veredas, destaca-se o perifíton. Este representa um elo físico entre o substrato e a água circundante, onde ocorrem processos internos (autotróficos e heterotróficos) e trocas com o meio, como também com o próprio substrato, funcionando, portanto, como um microcosmo (Sládečková, 1962; Wetzel, 1983; Weitzel, 1979). Como constituintes do complexo perifítico, as algas são crucialmente importantes para o funcionamento destes ecossistemas, uma vez que são produtoras primárias.

As algas perifíticas possuem atributos que as tornam indicadores ideais da qualidade da água, como hábito aderido, curtos ciclos de vida, alta riqueza e abundância de espécies em uma área pequena e como primeiro elo entre os componentes físicos e químicos da água e a comunidade biótica da cadeia alimentar (Lowe \& Pan, 1996; McCormick \& Stevenson, 1998). Apesar da importância das veredas como um ecossistema neotropical, bem como das algas, estudos sobre esses microrganismos são incipientes, destacando apenas Menezes (1986) para a taxonomia de algas planctônicas e Dunck et al. (2013) para ecologia de algas perifíticas.

Estudos enfocando tais grupos de algas têm demonstrado maiores valores de diversidade de espécies relacionados à ambientes com impactos intermediários (Stevenson, 1996; Biggs et al., 1998). De acordo com este pressuposto e com o apontado por Dunck et al. (2013), no qual áreas úmidas (Veredas) com acentuado desmatamento das margens (maior disponibilidade de luz) e maiores cargas de nutrientes (via alóctone) apresentaram maior riqueza, densidade e biovolume de algas epifíticas, este estudo buscou testar a hipótese de que Veredas situadas em áreas urbanas e agropecuária abrigam maior riqueza taxonômica, diversidade e densidade total de algas perifíticas, em função da ação antrópica. Para isso, nós investigamos: a) a diferenciação na composição da diversidade de espécies e densidade total das comunidades de algas perifíticas em Veredas com diferentes impactos; e b) quais variáveis abióticas que mais influenciaram a estrutura das comunidades nestes ambientes.

\section{Material e Métodos}

\section{ÁREAS DE ESTUDO}

Foram selecionadas seis Veredas, distribuídas nos Municípios de Caldas Novas, Catalão, Ipameri e Morrinhos (Figura 1; Tabela 1), todas situadas na região sudeste do Estado de Goiás.

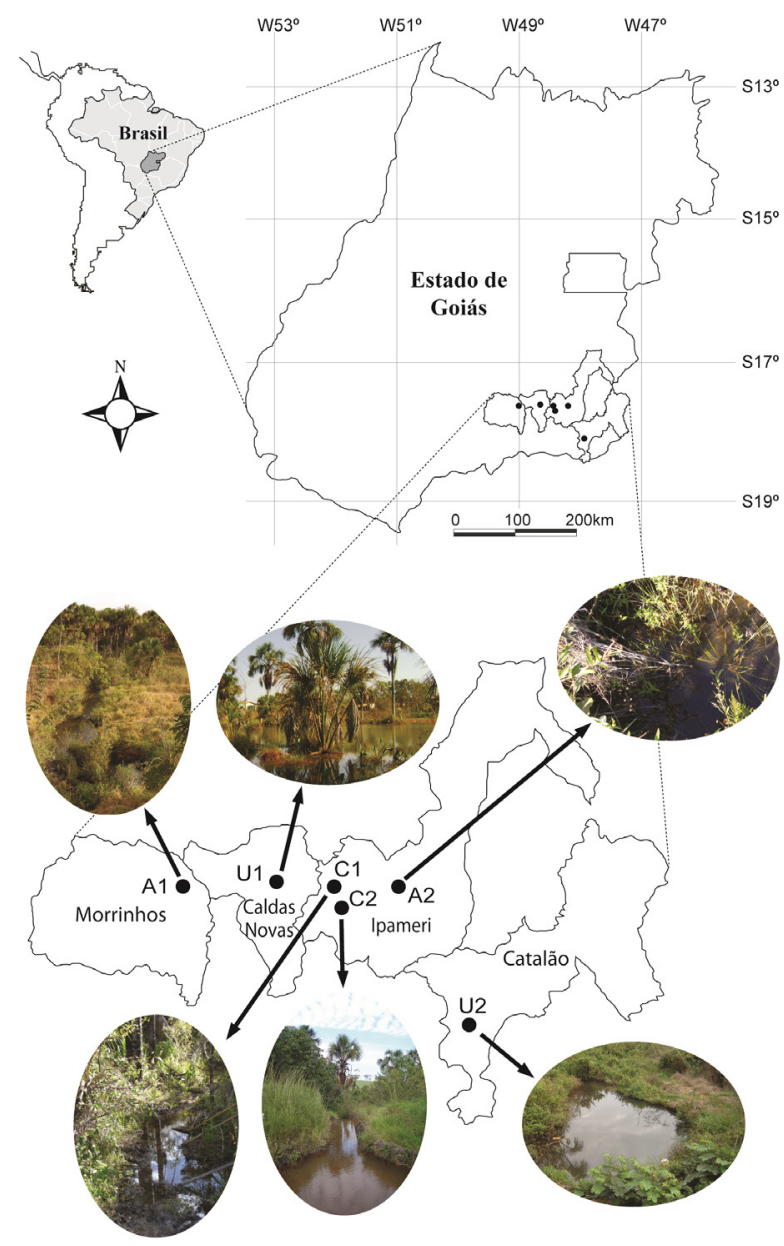

Figura 1 - Mapa com a localização das Veredas nos Municípios da região sudeste, Estado de Goiás. 
Tabela 1 - Informações das seis Veredas amostradas em 2008, 2009 e 2010, na região sudeste do Estado de Goiás. C1, C2 = conservadas; U1, U2 = urbanas; A1, A2 = agropecuária.

\begin{tabular}{cccc}
\hline Veredas & Município & Coordenadas geográficas & Cobertura vegetal \\
\hline A1 & Morrinhos & $17043,412^{\prime} \mathrm{S}-48^{\circ} 57,802^{\prime} \mathrm{W}$ & $<50$ m de mata ciliar \\
A2 & Ipameri & $17043,771^{\prime} \mathrm{S}-48^{\circ} 12,283^{\prime} \mathrm{W}$ & $<50$ m de mata ciliar \\
C1 & Ipameri & $17044,589^{\prime} \mathrm{S}-48^{\circ} 28,489^{\prime} \mathrm{W}$ & $>50$ m de mata ciliar \\
C2 & Ipameri & $17047,525^{\prime} \mathrm{S}-48^{\circ} 25,356^{\prime} \mathrm{W}$ & $>50$ m de mata ciliar \\
U1 & Caldas Novas & $17043,067^{\prime} \mathrm{S}-48^{\circ} 36,310^{\prime} \mathrm{W}$ & $<50$ m de mata ciliar \\
U2 & Catalão & $18^{\circ} 11,392^{\prime} \mathrm{S}-47^{\circ} 57,116^{\prime} \mathrm{W}$ & $<50$ m de mata ciliar \\
\hline
\end{tabular}

Fonte: Dunck et al. (2013).

Em Goiás existem dois períodos sazonais de precipitação: uma estação de seca, de maio a outubro, com nenhuma precipitação de junho a julho; e outra estação chuvosa, de novembro a abril (Figura 2).

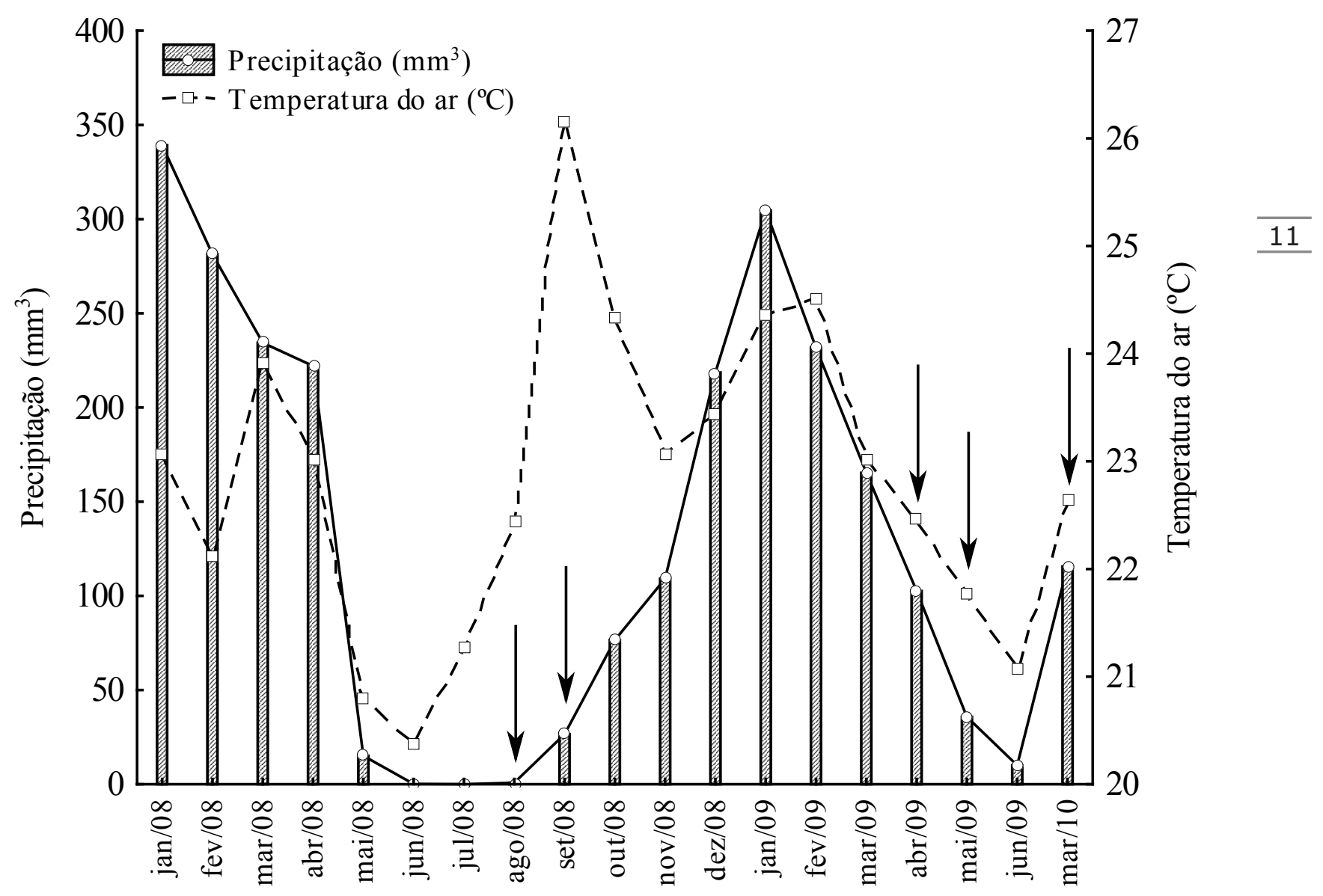

Figura 2 - Precipitação acumulada e temperatura do ar na região sudeste do Estado de Goiás (Caldas Novas, Catalão, Ipameri e Morrinhos), de janeiro/2008 a março/2010. As setas indicam o mês da amostragem dos dados bióticos e abióticos. 
As Veredas amostradas foram classificadas como conservadas ( $\mathrm{C} 1$ e $\mathrm{C} 2$ ), impactadas por agropecuária ( $A 1$ e $A 2$ ) e impactadas por áreas urbanas (U1 e U2). As Veredas A1 e A2 estão situadas em área de fazenda. A primeira, interrompida pela estrada (GO-147), assim como a segunda possuem margens desmatadas, região de plantio de algodão e soja, além da presença de gado. As Veredas U1 e U2, localizadas em área urbana de Caldas Novas e Catalão, respectivamente, encontram-se bastante alteradas, devido à movimentação de veículos, presença de animais, represamentos, além de estarem também interrompidas por estradas. Ambas as Veredas U1 e U2 recebem esgotos de condomínios adjacentes e têm lixo nas margens. As Veredas C1 e C2 estão dentro de uma área de fazenda e em estado mais preservado com relação as anteriores, sendo que C2 está situada em área de Cerrado Sensu Stricto.

Em relação ao estado trófico, para o índice proposto por Lamparelli (2004) que considera as concentrações de fósforo total, todas as seis Veredas foram classificadas como oligotróficas. Para o índice proposto por Dodds et al. (1997) que considera as concentrações de nitrogênio total, as Veredas foram classificadas como mesotróficas (U1 e U2), eutróficas (A1, A2 e C2) e hipereutróficas (C1) (Dunck et al., 2013).

\section{AMOSTRAgEM E ANÁLISE LABORATORIAL}

As amostragens foram realizadas em cinco campanhas nas seis Veredas. A primeira amostragem foi realizada de 30/08 a 01/09 de 2008; a segunda de 24 a 25/09/08; a terceira de 25 a 26/04/09, a quarta de 27 a 28/05/09 e a última no período de 11 a 12/03/2010.

O perifíton foi coletado em substrato natural (gramíneas) nas seis Veredas, sendo um pecíolo para cada amostra qualitativa e quantitativa. O material perifítico foi removido dos substratos com auxílio de espátula e jatos d'água destilada, e transferidos para frascos $(100 \mathrm{~mL})$. O material para análise quantitativa foi fixado com solução de lugol acético $0,5 \%$ e para amostras qualitativas com solução de Transeau.

As variáveis abióticas amostradas foram: oxigênio dissolvido $\left(\mathrm{mg}^{\mathrm{L}} \mathrm{L}^{-1}\right), \mathrm{pH}$, temperatura da água $\left({ }^{\circ} \mathrm{C}\right)$, condutividade elétrica $\left(\mu \mathrm{m} . \mathrm{S}^{-1}\right)$ e turbidez (NTU) utilizando uma sonda de análise multi-parâmetro Horiba modelo U-21. Amostras de água foram coletadas para determinação das concentrações de nutrientes em $\mu \mathrm{g} \mathrm{L}^{-1}$ (fósforo e nitrogênio, nitrato e amônia) e armazenadas em freezer no Laboratório de Análise e Gerenciamento Ambiental de Recursos Hídricos (LAMA$\mathrm{RH})$, da Universidade Federal de Goiás (UFG), até serem destinadas ao processamento. As análises de nutrientes foram realizadas de acordo com Standard Methods da AMERICAN PUBLIC HEALTH ASSOCIATION (APHA, 2005). Os dados climatológicos de temperatura do ar e precipitação acu- mulada foram cedidos pela Secretaria de Ciência e Tecnologia do Estado de Goiás (SECTEC/GO).

A análise quantitativa das 60 amostras de material perifítico ( $n=2$, para cada amostra) foi realizada em microscópio invertido, através do método de Utermöhl (1958), em campos aleatórios até atingir uma estabilização da curva (de rarefação de espécies) e um total de 100 indivíduos da espécie mais comum (Bicudo, 1990). A densidade das algas perifíticas foi estimada segundo a equação de Ros (1979) e os valores da densidade convertidos para unidade de área do substrato $\left(\mathrm{cm}^{2}\right)$.

A identificação das algas foi fundamentada na literatura clássica, com destaque para Croasdale \& Flint (1986, 1988), Dillard (1990, 1991), Förster (1982), Komárek \& Anagnostidis (1999, 2005), Krammer \& Lange-Bertalot (1986, 1988, 1991), Patrick \& Reimer (1966, 1975), Prescott (1982), Prescott et al. $(1981,1982)$ entre outras específicas e regionais. Foi seguido a recomendação de Bicudo \& Menezes (2006) para o sistema de classificação das algas.

\section{ANÁLISE dOS DADOS}

A Análise de Componentes Principais (PCA) foi usada para verificar a variação espacial e temporal dos ambientes quanto às variáveis limnológicas $(\mathrm{pH}$, temperatura da água, condutividade elétrica, turbidez, oxigênio dissolvido, fósforo total, nitrogênio total, amônia e nitrato). Para esta análise as variáveis, exceto o pH, foram log-transformadas $[\log (x+1)]$. O critério utilizado para reter os eixos para interpretação foi o modelo de Broken-Stick (Jackson, 1993).

A diversidade de algas perifíticas das seis Veredas foi determinada pelo índice de Shannon-Wiener. A análise de espécies indicadoras (Indval) (Dufrêne \& Legendre, 1997) foi utilizada para avaliar a existência de espécies possivelmente relacionadas aos tipos de impactos nos ambientes estudados. Para isso, utilizamos a matriz de densidade dos táxons dominantes e abundantes $(\log x+1)$ e o impacto das Veredas como fator. Este método baseia-se na comparação das abundâncias relativas (especificidade) e frequência de ocorrências (fidelidade) dos táxons em diferentes conjuntos de ambientes. O valor de indicação (IV), que varia de 0 a 100, será máximo quando os indivíduos de uma determinada espécie apresentarem alta especificidade e alta fidelidade a todos os ambientes de um determinado grupo especificado a priori. Esta análise é testada através do teste de Monte Carlo com 999 aleatorizações e valor de $p<0,05$. Todas as análises foram realizadas através do programa PC-ORD 5.15 (McCune \& Mefford, 2006).

\section{Resultados}

Ao todo, foram amostrados 366 táxons de algas perifíticas distribuídos em 15 classes, em todas as 
amostragens nas seis Veredas: Bacillariophyceae, Chlorophyceae, Chrysophyceae, Cryptophyceae, Cyanophyceae, Dinophyceae, Euglenophyceae, Fragilariophyceae, Klebsormidiophyceae, Oedogoniophyceae, Rhodophyceae, Trebouxiophyceae, UIvophyceae, Xanthophyceae e Zygnematophyceae.

Em relação a densidade algal, nas Veredas situadas em área de agropecuária, os maiores valores ocorreram em A1, enquanto nas conservadas em C2, especialmente em março de 2010 (Figura 3). Já nas Veredas urbanas os maiores valores foram registrados para $U 2$, principalmente em maio de 2009 (1295 ind.103. $\mathrm{cm}^{-2}$ ) (Figura 3).

As maiores densidades de algas perifíticas (mais de $100 \mathrm{mil}$ ind. $\mathrm{cm}^{-2}$ ) foram registradas para Cyanophyceae. Nas Veredas impactadas por agropecuária, os maiores valores de densidade de Cyanophyceae foram registrados em agos- to/2008 e abril e maio/2009; nas urbanas em abril/2009 e maio/2010; e nas Veredas conservadas em março/2010 (Figura 3).

Já para as Bacillariophyceae (diatomáceas), os maiores valores (mais de 45 mil ind $\mathrm{cm}^{-2}$ ) para as Veredas urbanas foram registrados em agosto e setembro/2008 e em abril e maio/2009; para as impactadas por agropecuária em abril/2009; e para as conservadas em março/2010 (Figura 3).

Vale destacar a representatividade de Zygnematophyceae, as desmídias (mais de 50 mil ind. $\mathrm{cm}^{-2}$ ), para Veredas urbanas em abril/2009 e para Veredas conservadas em março/2010 (Figura 3). Com relação a Oedogoniophyceae (mais de 40 mil ind. $\mathrm{cm}^{-2}$ ), os maiores valores de densidade foram registradas em março/2010 para Veredas conservadas e em setembro/2009 para as urbanas (Figura 3).
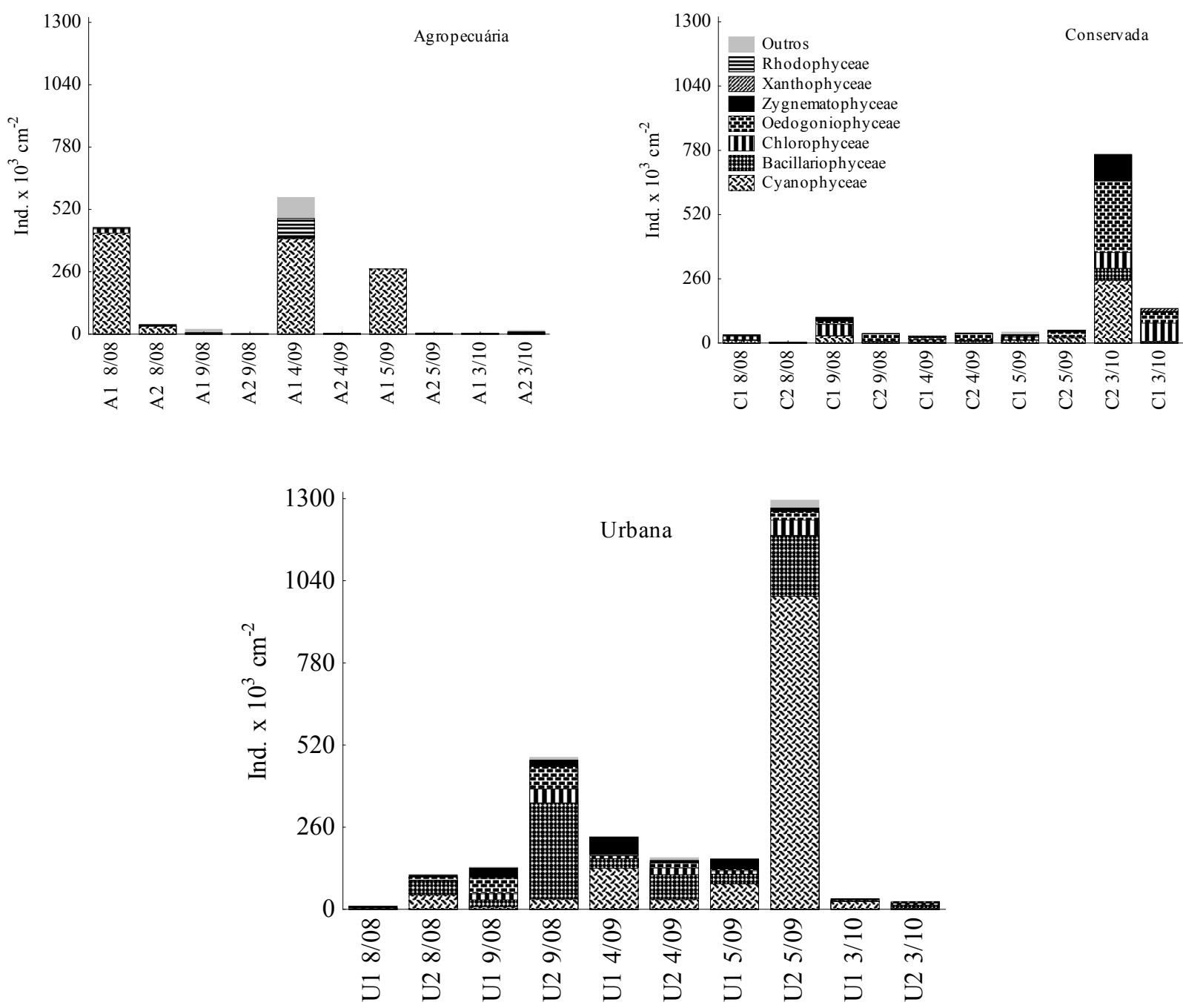

Figura 3 - Densidade geral (indiv. $10^{3} . \mathrm{cm}^{-2}$ ) por classes de algas perifíticas registradas nas Veredas em área de Agropecuária, conservada e urbana, nos anos de 2008, 2009 e 2010. Outras classes: Chrysophyceae, Cryptophyceae, Dinophyceae, Euglenophyceae, Fragilariophyceae, Klebsormidiophyceae, Ulvophyceae, Xanthophyceae. 
Os maiores valores de diversidade de Shannon e riqueza taxonômica foram verificados para as Veredas urbanas, especialmente no mês de setembro. A equitabilidade foi maior nas conservadas e impactadas por agropecuária (Figura 4).
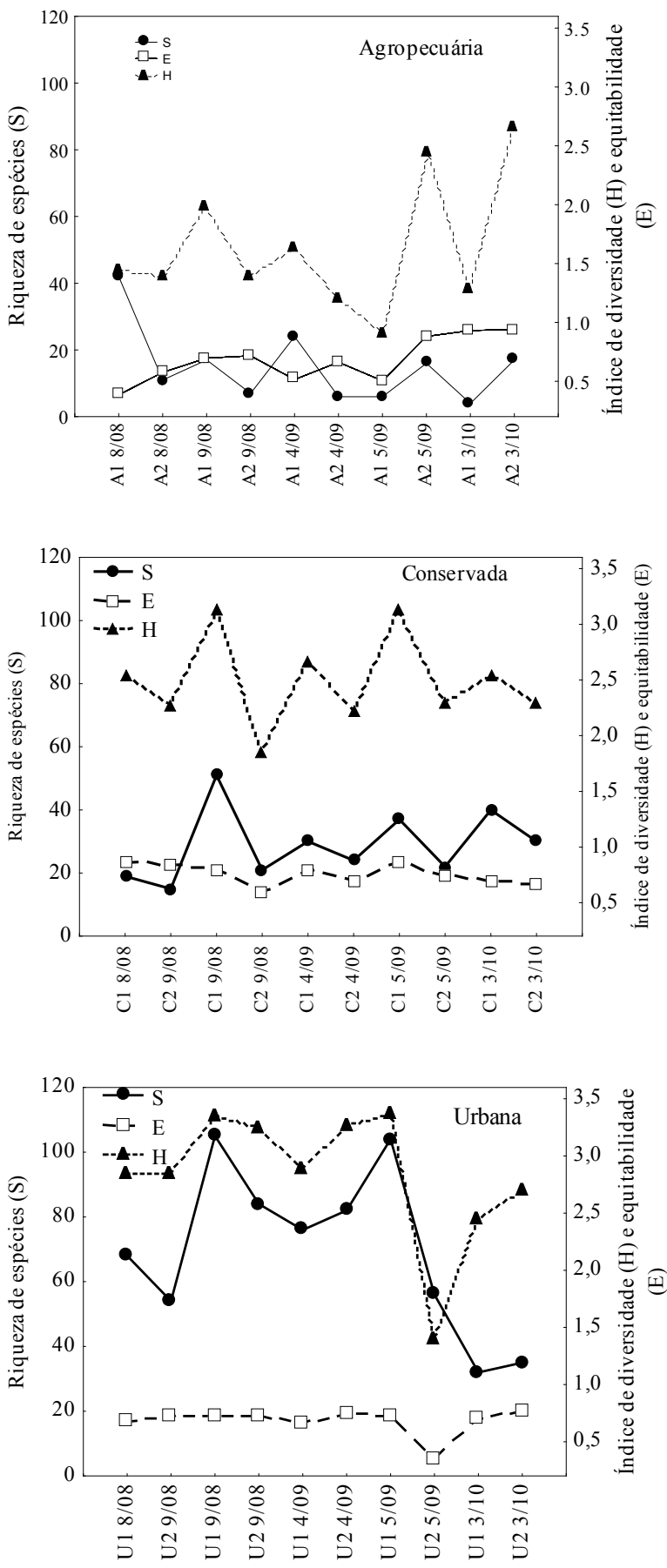

Figura 4 - Índice de diversidade Shannon-Wiener $\left(\mathrm{H}^{\prime}\right)$, riqueza taxonômica (S) e equitabilidade (E) para as comunidades de algas perifiticas registrado em seis Veredas da região sudeste do Estado de Goiás, nos anos de 2008, 2009 e 2010.
A análise de espécies indicadoras (Indval) revelou que dos 15 táxons com abundância relativa e frequência de ocorrência relacionadas aos tipos de impactos das Veredas estudadas, 11 estão presentes em Veredas de áreas de agropecuária e urbana (Tabela 2). Veredas em área urbana foram relacionadas com seis espécies pertencentes ao grupo das diatomáceas, enquanto Veredas em área de agropecuária tiveram três espécies associadas de Zygnematophyceae dos cinco táxons (Tabela 2).

Tabela 2 - Lista de táxons indicadores dos grupos de Veredas sob diferentes impactos; grupo $\mathrm{A}=$ Veredas situadas em área de agropecuária; grupo $\mathrm{C}=$ Veredas em áreas conservadas; grupo $\mathrm{U}=\mathrm{Ve}-$ redas em áreas urbanas.

\begin{tabular}{clcc}
\hline Grupo & Táxons & IV & p \\
\hline A & Cosmarium cf. brasiliense & 38.6 & 0.0452 \\
A & Cosmarium cf. punctulatum & 55.9 & 0.0102 \\
A & Haplotaenium minutum & 46.8 & 0.0128 \\
A & Homeothrix sp. & 50.0 & 0.0056 \\
A & Navicula sp. & 49.0 & 0.0238 \\
C & Chaetosphaeridium sp. & 58.6 & 0.0058 \\
C & Chroococcus sp. & 38.9 & 0.0470 \\
C & Oedogonium sp.2 & 56.2 & 0.0430 \\
C & Oedogonium sp.4 & 84.1 & 0.0140 \\
U & Achnanthidium cf. minutissimum & 39.8 & 0.0242 \\
U & Encyonema cf. neomesianun & 37.2 & 0.0400 \\
U & Encyonema cf. neogracile & 40.0 & 0.0242 \\
U & Fragilaria cf. capucina & 45.2 & 0.0178 \\
U & Gomphonema gracile & 49.8 & 0.007 \\
U & Gomphonema parvulum & 55.3 & 0.0326 \\
\hline
\end{tabular}

Os resultados da Análise de Componentes Principais (PCA) demonstram $54,7 \%$ da variância acumulada nos dois primeiros eixos (Tabela 3; Figura 5), tendo o período de coleta o principal fator de diferenciação entre as unidades amostrais. As Veredas do mês de maio/2009 apresentaram $\mathrm{pH}$ mais ácido e maiores valores de amônia, nitrato e nitrogênio total e menor condutividade elétrica, enquanto que as Veredas amostradas em março/2010 (período de chuvas) relacionaram-se aos maiores valores de temperatura da água, $\mathrm{pH}$ mais próximo a neutralidade e turbidez mais elevada (Figura 5). As Veredas amostradas em 2008 (período de seca) foram separadas das demais por terem menor concentração de fósforo total e maior condutividade (Figura 5), com valores variando de 6 a $30 \mu \mathrm{m} . \mathrm{S}^{-1}$. 
Tabela 3 - Valores de correlação obtidos através da Analise de Componentes Principais (PCA), com dados abióticos e bióticos para seis Veredas, nos anos de 2008, 2009 e 2010.

\begin{tabular}{lcc}
\hline Variáveis & Eixo $\mathbf{1}$ & Eixo 2 \\
\hline pH & $-0,0200$ & $-0,7620$ \\
Temperatura da água & $-0,0327$ & $-0,8084$ \\
Condutividade Elétrica & 0,6044 & $-0,2599$ \\
Turbidez & $-0,7683$ & $-0,4470$ \\
Oxigênio Dissolvido & $-0,3539$ & 0,1198 \\
Fósforo Total & 0,0904 & 0,2496 \\
Nitrogênio Total & $-0,7790$ & 0,0920 \\
Amônia & $-0,9092$ & 0,1372 \\
Nitrato & $-0,8950$ & 0,0132 \\
\% acumulada nos eixos & 36,9 & 17,8 \\
Autovalores & 3,325 & 1,606 \\
Broken-stick & 2,829 & 1,829 \\
\hline
\end{tabular}

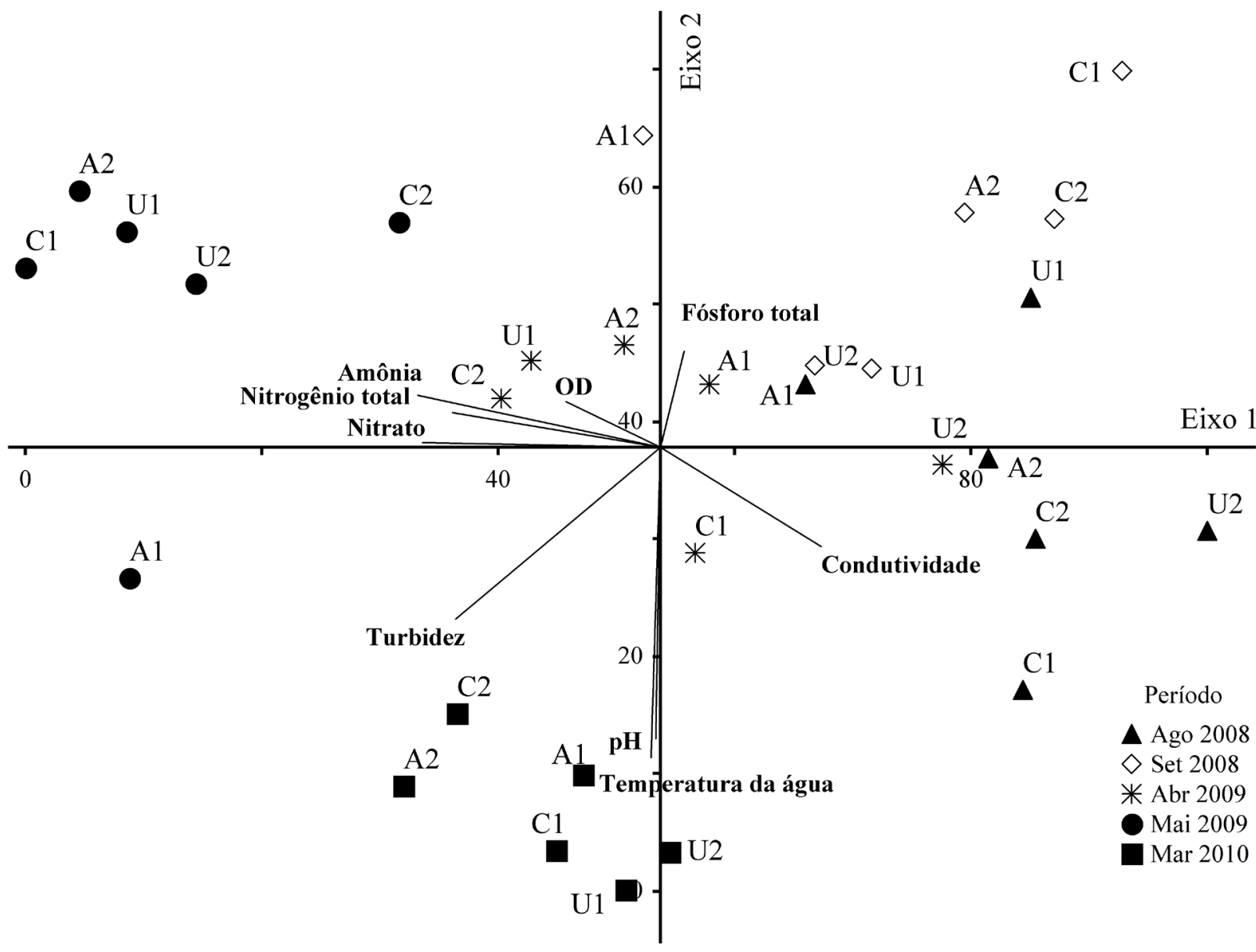

Figura 5 - Análise dos componentes principais (PCA) para as variáveis abióticas e bióticas das seis Veredas da região sudeste do Estado de Goiás, em 2008, 2009 e 2010. A1 e A2 = Veredas impactadas por agropecuária; C1 e C2 = Veredas conservadas; U1 e U2 = Veredas impactadas por áreas urbanas. 


\section{Discussão}

Os resultados deste estudo demonstraram que o impacto antrópico desempenhou um papel importante na estruturação das comunidades de algas perifíticas. Porém, a sazonalidade foi determinante na diferenciação dos habitats entre as Veredas (como evidenciado pela PCA), resultando nos padrões de ocorrência e densidade das espécies de algas.

O aumento de nutrientes via alóctone e a remoção de sedimentos são influenciados pela precipitação, principalmente em ambientes impactados, que faltam vegetação marginal (Fernandes \& Esteves, 2003). Com isso, os processos ambientais de cada habitat exercem uma ação direta no desenvolvimento da comunidade ficoperifítica (Weitzel, 1979; Casco \& Toja, 1994), bem como promovem uma maior heterogeneidade de microhabitats, o que facilita a invasão de propágulos e de novos indivíduos (Algarte et al., 2013).

Nesse estudo, os maiores valores de densidade e riqueza taxonômica de algas perifíticas em Veredas impactadas estiveram relacionados com os nutrientes, turbidez e temperatura da água. Maiores concentrações de nutrientes (Baker et al., 2009), represamento da água e maior incidência de radiação solar (Tuji, 2000) são fatores importantes na estruturação e estabelecimento das algas perifíticas nos substratos (Dunck et al. 2013). Porém, a temperatura influencia na dominância de certas espécies de algas, alterando a composição e diversidade, mais do que a disponibilização de nutrientes (Murakami \& Rodrigues, 2009), além de determinar a distribuição geográfica e interações de competição e trofia (DeNicola, 1996; Butterwick et al., 2005; Imai et al., 2009).

$\mathrm{Na}$ maioria das Veredas, especialmente naquelas situadas em áreas urbanas, as diatomáceas e desmídias foram predominantes. As diatomáceas são compostas por inúmeras espécies oportunistas (r-estrategistas, Biggs, 1995), que possuem capacidade mais rápida de reprodução em ambientes impactados e de elevada disponibilidade de nutrientes (Carrick \& Steinman, 2001). Diatomáceas produzem pedúnculos e poros mucilaginosos que facilitam a adesão a substratos (Stoermer \& Smol, 1999; Round et al., 2000; Winter \& Duthie, 2000; Molino \& Wetherbee, 2008). Ainda, possuem clorofila-c que proporciona alta adaptabilidade em ambientes túrbidos (Reynolds, 1995). No presente estudo, as Veredas impactadas tiveram turbidez elevada, que pode ter beneficiado este grupo de algas.

Entre as diatomáceas, Gomphonema gracile Ehrenberg é destacada como tolerante à variação de $\mathrm{pH}$ e com preferência por águas com menor concentração de nutrientes (Patrick \& Reimer, 1966; Potapova \& Charles, 2007), e classificada dentro do grupo de espécies menos tolerantes à poluição (Salomoni et al., 2006). No entanto, outros estudos indicam G. gracile como tolerante à poluição (Van Dam et al., 1994; Lobo et al.,
2002; Stenger-Kovács et al., 2007). Neste estudo, G. gracile foi indicador de Veredas impactadas urbanas, com condições oligotróficas em relação ao fósforo total e mesotróficas para nitrogênio total (Dunck et al., 2013). Esses resultados contraditórios podem ser explicados pela variabilidade morfológica deste táxon, podendo, ainda, ser composto por um complexo de espécies com necessidades ecológicas distintas (Tremarin et al., 2009).

A predominância de G. parvulum assim como de Achnanthidium cf. minutissimum (Kützing) Czarnecki, Encyonema cf. neogracile Krammer, Encyonema cf. neomesianum, Fragilaria cf. capucina Desmaziéres, Gomphonema complexo gracile, G. parvulum, foi relacionada com as condições oligotróficas e mesotróficas das Veredas urbanas. Dessa maneira, essas espécies de algas podem ser consideradas indicadoras desses locais.

As desmídias e as diatomáceas são compostas por táxons unicelulares não flagelados. Organismos unicelulares têm maior aptidão para proliferar mais rapidamente; enquanto organismos multicelulares, que em função de estruturas vegetativas e reprodutivas mais complexas, exigem maior tempo de desenvolvimento e são mais lentos no processo de proliferação (Hillebrand \& Sommer, 2000). Isso, provavelmente, também explica a maior riqueza de organismos unicelulares não flagelados em nosso estudo.

As células basais das algas filamentosas (Oedogoniophyceae) facilitam sua permanência ao substrato, além de desenvolverem-se preferencialmente em ambientes mais rasos e com temperatura entre 25 e $30^{\circ} \mathrm{C}$ (Dias, 1997). Desta forma, possivelmente tais condições propiciaram altos valores de indicação a Oedogonium sp. 2 e Oedogonium sp. 4 em ambas as Veredas C1 e C2.

Estudos sobre perifíton em lagoas têm demonstrado que o aumento na densidade de Cyanophyceae esta relacionado com a estabilidade térmica do ambiente (Murakami \& Rodrigues, 2009). Nesse trabalho, ambientes lênticos e rasos como as Veredas propiciam uma estabilidade maior em relação à temperatura da água e assim podem beneficiar a abundância de cianofíceas. Outros motivos para a predominância deste grupo de organismos deve-se ao fato dos indivíduos se multiplicarem assexuadamente (Reviers, 2006), ao metabolismo fotossintético menos exigente em comparação a outros grupos algais, bem como as estratégias em assimilar e estocar nutrientes (Whitton \& Potts, 2002). Com isso, podem se adaptar as mudanças e flutuações ambientais independentemente dos recursos combinados de nitrogênio.

Podemos concluir que os maiores valores de riqueza taxonômica, diversidade e densidade de algas perifíticas em Veredas impactadas devem-se a fatores proporcionados pela ação antrópica (desmatamentos e a maiores cargas de nutrientes via alóctone) e a sazonalidade. Estes fatores provocaram alterações nas características limnológicas dos ambientes aquáticos propiciando aumento na concentração de nutrientes e na turbidez. 
Assim, as diferenciações das condições ambientais das seis Veredas determinaram os padrões estruturais das comunidades de algas perifíticas, especialmente, no predomínio de Zygnematophyceae (três táxons indicadores de Veredas impactadas por agropecuária) e de Bacillariophyceae (seis táxons indicadores de Veredas urbanas).

\section{Agradecimentos}

À Coordenação de Aperfeiçoamento de Pessoal De Nível Superior (CAPES) pela concessão de bolsa Prodoc às duas primeiras autoras e bolsa de doutorado para a terceira autora. Ao Programa de Pós-Graduação em Ciências Ambientais da Universidade Federal de Goiás (CIAMB-UFG) pelo financiamento parcial do estudo (Processo CAPES no 23038.040928/2008-61) e ao Jaime Luiz Lopes Pereira pela confecção do mapa da área de estudo.

\section{ReferênCIAS}

Algarte, V.M., N.S. Siqueira \& L. Rodrigues. 2013. Desiccation and recorevy of periphyton biomass and density in a subtropical lentic ecosystem. Acta Scientiarum. Biological Sciences 35(3): 2013.

APHA. 2005. American Public Health Association - APHA; American water works association - AWWA, water pollution control federation WEF. Standard Methods for the Examination of Water and Wastewater. $21^{\circ} \mathrm{Ed}$. Washington, D. C., EUA.

Baker, M.A., G. Guzman \& J.D. Ostermiller. 2009. Differences in nitrate uptake among benthic algal assemblages in a mountain stream. Journal of the North American Benthological Society 28: 24-33.

Bicudo, D.C. 1990. Considerações sobre metodologias de contagem de algas do perifiton. Acta Limnologica Brasiliensia 3: 459-475.

Bicudo, C.E.M. \& M. Menezes. 2006. Gêneros de algas de águas continentais do Brasil (Chave de identificação e descrições). RiMa, São Carlos.

Biggs, B.J.F. 1995: The contribution of flood disturbances, catchment geology and land use to the habitat template of periphyton in stream ecosystems. Freshwater biology 33: 419-438.

Biggs, B.J.F., R.J. Stevenson \& R.L. Lowe. 1998. A habitat matrix conceptual model for stream periphyton. Archiv für Hydrobiologie 143: 21-56.
Butterwick, C., S.I. Heaney \& J.F. Talling. 2005. Diversity in the influence of temperature on the growth rates of freshwater algae, and its ecological relevance. Freshwater Biology 50: 291-300.

Carrick H.J. \& A.D. Steinman. 2001. Variation in periphyton biomass and species composition in Lake Ockeechobee, Florida (USA): distribution of algal guilds along environmental gradients. Archiv für Hydrobiologie 152: 411-438.

Casco, M.A. \& J. Toja. 1994. The distribution and interaction of algal communities in reservoir. Archiv für Hydrobiologie Beiheft Ergebnisse der Limnologie 40: 85-96.

Croasdale, H. \& E.A. Flint. 1986. Flora of New Zealand: Freshwater algae, Chlorophyta, Desmids. Wellington, New Zealand, v. I, 27 plates, 132p.

Croasdale, H. \& E.A. Flint. 1988. Flora of New Zealand: Freshwater algae, Chlorophyta, Desmids. Christchurch, New Zealand, v. II, 33 plates, $146 \mathrm{p}$.

DeNicola, D.M. 1996. Periphyton responses to temperature at different ecological levels. Pp. 149-181 In: Stevenson, R.J., M.L. Bothwell, \& R.L. Lowe (eds). Algal ecology: freshwater benthic ecosystems. Academic Press, San Diego, California.

Dias, I.C.A. 1997. Chlorophyta filamentosas da reserva biológica de Poço das Antas, Município de Silva Jardim, Rio de Janeiro: Taxonomia e aspectos ecológicos. Tese de Doutorado. - Instituto de Biociências, Universidade de São Paulo, São Paulo.

Dillard, G.E. 1990. Freshwater Algae of the Southeastern United States. Part 3. Chrolophyceae: Zygnematales: Mesotaeniaceae and Desmidiaceae (Section 1). Berlin; Stuttgart: J. Cramer. 172p., il., 51 planches. (Bibliotheca phycologica, band 85).

Dillard, G.E. 1991. Freshwater Algae of the Southeastern United States. Part 4. Chrolophyceae: Zygnematales: Desmidiaceae (Section 2). Berlin; Stuttgart: J. Cramer. 205p., il., 52 planches. (Bibliotheca phycologica, band 89).

Dodds, WK., V.H. Smith \& B. Zander. 1997. Developing nutrient targets to control benthic chlorophyll levels in streams: a case study of the Clark Fork River. Water Research 31: 1738-1750. 
Dufrêne, M. \& P. Legendre. 1997. Species assemblages and indicator species the need for a flexible asymmetrical approach. Ecological Society of America 67(3): 345-366.

Dunck, B., I.S. Nogueira \& S. A. Felisberto. 2013. Distribution of epiphytic algae in wetlands (Palm swamps, Cerrado), Brazil. Brazilian Journal of Biology 73(2): 331-346.

Eiten, G. 1972. The Cerrado vegetation of Central Brazil. The Botanical Review 38: 201-341.

Eiten, G. 1994. Vegetação, p. 17-73. In: M. N. Pinto (ed.), Cerrado: caracterização, ocupação e perspectivas. Editora da Universidade de Brasília, Brasília.

Fernandes, V.O. \& F.A. Esteves. 2003. The use of indices for evaluating the periphytic community in two kinds of substrate in Imboassica Lagoon, Rio de Janeiro, Brazil. Brazilian Journal of Biology 63(2): 233-243.

Ferreira, I. M. 2003. O afogar das Veredas: uma análise comparativa espacial e temporal das Veredas do Chapadão de Catalão (GO). Tese de Doutorado. Programa de Pós-Graduação em Geografia. Universidade Estadual Paulista, Rio Claro, SP.

Förster, K. 1982. Conjugatophyceae: Zygnematales und Desmidiales (excl. Zygnemataceae), p. 1-543. In: G. Huber-Pestalozzi (ed.). Das Phytoplankton des Süsswassers: Systematik und Biologie. Schweizerbart'sche Verlagsbuchhandlung, Stuttgart.

Hillebrand, H. \& U. Sommer. 2000. Diversity of benthic microalgae in response to colonization time and eutrophication. Aquatic Botany 67: 221-223.

Imai, H., K.H. Chang, M. Kusaba \& S.I. Nakano. 2009. Temperature-dependent dominance of Microcystis (Cyanophyceae) species: M. aeruginosa and M. wesenbergii. Journal of Plankton Research 31(2): 171-178.

Jackson, D.A. 1993. Stopping rules in principal components analysis: a comparison of heuristical and statistical approaches. Ecology 74: 2204-2214.

Komárek, J. \& K. Anagnostidis. 1999. Cyanoprocaryota 1 - Chroococcales, p. 1-548. In: H. Ettl, J. Gerloff, H. Heynig \& D. Mollenhauer (eds.) SüBwasserflora von Mitteleuropa. Gustav Fisher, Stuttgart.

Komárek, J. \& K. Anagnostidis. 2005. Cyanoprocaryota 2 - Oscillatoriales, p. 1-759. In:
H. Ettl, J. Gerloff, H. Heynig \& D. Mollenhauer (eds.) SüBwasserflora von Mitteleuropa. Gustav Fisher, Stuttgart.

Krammer, K. \& H. Lange-Bertalot. 1986. Bacillariophyceae: Naviculaceae, p. 1-876, $2^{\circ}$ vol. In: H. Ettl, J. Gerloff, H. Heynig \& D. Mollenhauer (eds.), Süsswasser flora von Mitteleuropa. Gustav Fischer, Stuttgart.

Krammer, K. \& H. Lange-Bertalot. 1988. Bacillariophyceae: Bacillariaceae, Epithemiaceae, Surirellaceae, p. 1-596, $2^{\circ}$ vol. 2/2. In: H. Ettl, J. Gerloff, H. Heynig \& D. Mollenhauer (eds.), Süsswasser flora von Mitteleuropa. Gustav Fischer, Stuttgart.

Krammer, K. \& H. Lange-Bertalot. 1991. Bacillariophyceae: Centrales, Fragilariaceae, Eunotiaceae, p. 1-576, $2^{\circ}$ vol. 3. In: H. Ettl, J. Gerloff, H. Heynig \& D. Mollenhauer (eds.), Süsswasser flora von Mitteleuropa. Gustav Fischer, Stuttgart.

Lamparelli, M.C. 2004. Grau de trofia em corpos d'água do estado de São Paulo: avaliação dos métodos de monitoramento. São Paulo: Universidade de São Paulo. 238 p. Tese de Doutorado em Ecologia Aplicada.

Lima, S.C. 1991. A preservação das Veredas para manutenção do equilíbrio hidrológico dos cursos d'água, p. 204-218. In: Anais, Encontro Nacional de estudos sobre o meio ambiente. UEL/NEMA, Londrina.

Lobo, E.A., V.L.M. Callegaro \& E.P. Bender. 2002. Utilização de algas diatomáceas epilíticas como indicadoras da qualidade da água em rios e arroios da região hidrográfica do Guaíba, RS, Brasil. EDUNISC, Santa Cruz do Sul. 127p.

Lowe, R.L. \& Y. Pan. 1996. Benthic algal communities as biological monitors. In: Stevenson, R.J., M.L. Bothwell \& R.L. Lowe Algal Ecology: freshwater benthic ecosystems. New York: Academic Press. p. 705-739.

McCormick, P. V. \& R.J. Stevenson. 1998. Periphyton as a tool for ecological assessment and management in the Florida everglades. Journal of Phycology 34: 726-733.

McCune, B. \& M.J. Mefford. 2006. PC-ORD: multivariate analysis of ecological data. Version 5.14. Gleneden Beach, Oregon: MJM Software.

Menezes, M. 1986. Ficoflórula da Chapada dos Guimarães e arredores, Mato Grosso, Brasil: Euglenaceae pigmentadas (Euglenophyceae). Rickia 13: 87-95. 
Molino, P.J. \& R. Wetherbee. 2008. The biology of biofouling diatoms and their role in the development of microbial slimes. Biofouling 24 (5): 365-379.

Murakami, EA. \& L. Rodrigues. 2009. Resposta das algas perifíticas às alterações de temperatura e ao enriquecimento artificial de nutrientes em curto período de tempo. Acta Scientiarum. Biological Sciences 31(3): 273-284.

Patrick, R. \& C.W. Reimer. 1966. The diatoms of the United States, exclusive of Alaska and Hawaii. Livingstgon Company, Philadelphia. v. 1 , pt. 1 (Monography n.13), 688p.

Patrick, R. \& C.W. Reimer. 1975. The Diatoms of United States: exclusive of Alaska and Hawaii. Academy of Natural Sciences, Philadelphia, v.2, pt.1. (Monography n.13). 213p.

Potapova, M.G. \& D.F. Charles. 2007. Diatom metrics for monitoring eutrophication in rivers of the United States. Ecological Indicators 7: 48-70.

Prescott, G.W. 1982. ALGAE of de Western great lakes area. Koenigstein/W-Germany: Otto Koeltz Science Publishers.

Prescott, G.W., H.T. Croasdale, W.C. Vinyard \& C.E. M. Bicudo. 1981. A Synopsis of North American Desmids; Part II. Desmidiaceae: Placodermae. Section 3. P. 1-720. In: G.W. Prescott (ed.), Desmidiales. University of Nebraska Press.

Prescott, G.W., C.E.M. Bicudo \& W.C. Vinyard. 1982. A Synopsis of North American Desmids. Part II. Desmidiaceae: Placodermae. Section 4. P. 1-698. In: G.W. Prescott (ed.), Desmidiales. University of Nebraska Press.

Reviers, B. 2006. Biologia e filogenia das algas. Porto Alegre: Artmed, $280 \mathrm{p}$.

Reynolds, C.S. 1995. River plankton: the paradigm regained. In: Harper, D.M. \& A.J.D. Ferguson (eds.), The Ecological Basis for River Management. Wiley, Chichester, p. 161-174.

Ribeiro, J.F. \& B.M.T. Walter. 1998. Fitofisionomias do bioma Cerrado, p. 89-166. In: S.M. Sano \& S.P. Almeida (eds.), Cerrado: ambiente e flora. Embrapa-CPAC, Planaltina.

Ros, J. 1979. Prácticas de Ecologia. Ed. Omega, Barcelona. 181p.

Round, F.E., R.M. Crawford \& D.G. Mann. 2000. The Diatoms - Biology \& Morphology of the genera. Cambridge University Press.

Salomoni, S.E., O. Rocha, V.L.M. Callegaro \& E.A. Lobo. 2006. Epilithic diatoms as indi- cators of water quality in the Gravataí River, Rio Grande do Sul, Brazil. Hydrobiologia 559: 233-246.

Sládecková, A. 1962. Limnological investigation methods for the periphyton ("Aufwuchs") community. Revista de Botânica 28 (2): 286-350.

Stoermer, E.F. \& J.P. Smol. 1999. The Diatoms: Applications for the Environmental and Earth Sciences. Cambridge University Press, Cambridge.

Stenger-Kovács, C., K. Buczkó, E. Hajnal \& J. Padisák. 2007. Epiphytic, littoral diatoms as bioindicators of shallow lake trophic status: Trophic Diatom Index for Lakes (TDIL) developed in Hungary. Hydrobiologia 589: 141-154.

Stevenson, R.J. 1996. An introduction to algal ecology in freshwater benthic habitats. In: Stevenson, R.J., M.L. Bothwell \& R.L. Lowe (eds), Algal ecology: freshwater benthic ecosystems. Academic Press, San Diego. Pp. 3-30.

Tremarin, P.I., L.M. Bertolli, D.M. Faria, J.C. Costin \& T.A.V. Ludwig. 2009. Gomphonema Ehrenberg e Gomphosphenia LangeBertalot (Bacillariophyceae) do Rio Maurício, Paraná, Brasil. Biota Neotropica 9 (4): 111-130.

Tuji, A. 2000. The effect of irradiance on the growth of different forms of freshwater diatoms: implications for sucession in attached diatom communities. Journal of Phycology 36: 659-661.

Utermöhl, H. 1958. Zur Vervollkommung der quantitativen phytoplankton-methodik. Internationale Vereiningung fuer Theoretische und Angewandte Limnologie 9: 1-38.

Van Dam, H., A. Mertenes \& J. Sinkeldam. 1994. A coded checklist and ecological indicator values of freshwater diatoms from the Netherlands. Netherlands Journal of Aquatic Ecology 28: 117-133.

Weitzel, R.L. 1979. Periphyton measurements and application, p. 3-33. In: R.L. Weitzel (ed.) Methods and measurements of periphyton communities: a review. American Society for Testing and Materials, Philadelphia.

Wetzel, R.G. 1983. Opening remarks, p. 3-4. In: R.G. Wetzel (ed.) Periphyton of freshwater ecosystems. Developments in Hidrobiologia 17. The Hague: Dr. W. Junk. 
Winter, J.G. \& H.C. Duthie. 2000. Epilithic diatoms as indicators of stream total $\mathrm{N}$ and $\mathrm{P}$ concentration. Journal of the North American Benthological Society 19: 32-49.
Whitton, B.A. \& Potts. 2002. The Ecology of Cyanobacteria - Their diversity in time and space. Kluwer Academic Publisher, Dordrecht, The Netherlands, 669 pp.

Recebido em 31.I.2013

Aceito em 18.XI.2013 